\title{
Airborne exposure to wheat allergens: optimised elution for airborne dust samples
}

\author{
Jelena Bogdanovic, ${ }^{* a}$ Inge M. Wouters, ${ }^{a}$ Ingrid Sander, ${ }^{b}$ Eva Zahradnik, ${ }^{b}$ \\ Joanne Harris-Roberts (nee Elms), ${ }^{c}$ Maria-José Rodrigo, ${ }^{d}$ Susana Gómez-Ollés, ${ }^{d}$ \\ Dick J. J. Heederik ${ }^{a}$ and Gert Doekes ${ }^{a}$
}

Received 20th June 2006, Accepted 22nd August 2006

First published as an Advance Article on the web 31st August 2006

DOI: $10.1039 /$ b608770a

Well-validated methods for measuring airborne occupational allergens are essential for effective control and reduction of allergen exposures. For wheat flour allergens, specific immunoassays are available, but there is a need for optimisation and standardization of sample processing procedures. Wheat flour allergen elution and storage were studied using airborne dust samples collected in bakeries with a new parallel sampler. Forty-eight series of 9 parallel filters were subjected to extraction procedures varying in elution medium, shaking method, extraction vial, and centrifugation speed. Wheat allergens were measured with enzyme immunoassays, and the effect of various procedures evaluated by mixed regression analyses. The stability of the eluted allergens was assessed after storage for 20 days and 4 months at $-20{ }^{\circ} \mathrm{C}$, in the presence or absence of casein in the medium. Only the type of elution medium had significant effects on allergen recovery: addition of Tween-20 resulted in 3- to 100-fold increased levels, an effect that was most pronounced at low concentrations. Allergen levels in extracts were stable for at least 4 months at $-20{ }^{\circ} \mathrm{C}$, irrespective of the presence of casein in the medium. Addition of Tween-20 to the elution medium is essential for optimal extraction of wheat allergen. The recommended procedure further includes the use of conventional polystyrene tubes, simple shaking methods, and centrifugation after extraction. Wheat dust extracts in PBS-Tween can be stored frozen for at least 4 months, and addition of a stabilising protein is not required.

\section{Introduction}

Airborne dust of wheat flour (Triticum spp.) is an important sensitising agent causing occupational allergy and asthma in bakeries and flour mills. ${ }^{1-3}$ The water-soluble proteins in the flour are the most allergenic, as shown by their reactivity with IgE from sensitised bakery workers. ${ }^{4}$ Control measures to reduce allergen exposure may lead to a significantly decreased risk of occupational allergy in the bakery trade. ${ }^{5}$ Monitoring of airborne wheat allergens is essential to evaluate such control measures, but standardized methods are not available. Differences between laboratories in methods for dust sampling, extraction and wheat allergen measurements may induce large differences in reported allergen levels, as shown previously for other occupational allergens like fungal $\alpha$-amylase ${ }^{6}$ or rodent urinary proteins. ${ }^{7-10}$

As part of the European six-laboratory project MOCALEX (Measurement of Occupational Allergen Exposure), we investigated effects of differences in extraction protocols and immunoassay techniques ${ }^{11}$ on estimates of airborne wheat

\footnotetext{
${ }^{a}$ Institute for Risk Assessment Sciences, Division Environmental Epidemiology, Utrecht University, PO Box 80178, 3508 TD, The Netherlands.E-mail: j.bogdanovic@iras.uu.nl; Fax: +3130253 9499; Tel: + 31302531468

${ }^{b}$ Berufsgenossenschaftliches Forschungsinstitut für Arbeitsmedizin, Ruhr-Universität, Bochum, Germany

${ }^{c}$ Health and Safety Laboratory, Buxton, United Kingdom SK17 9JN

${ }^{d}$ University Hospital Vall D'Hebron, Barcelona, Spain
}

allergen levels. This paper describes the comparison of extraction methods using more than 400 filters with airborne flour dust sampled in bakeries and flour mills in four European countries. The following extraction parameters were investigated: elution medium, shaking method, type of extraction vial and centrifugation speed at which extracted proteins are separated from non-dissolved material. Furthermore, stability of wheat allergen after storage with and without a stabilising protein (casein) was assessed.

\section{Experimental}

\section{Parallel dust sampling}

A parallel sampler, enabling simultaneous collection of 10 identical samples of inhalable flour dust, was used to collect samples for comparison of elution procedures. The sampler was developed within the MOCALEX project according to a design published by Eduard et al., ${ }^{12}$ with modifications to fit PAS-6 sampling heads. Ten PAS-6 sampling heads ${ }^{13}$ were positioned in an annular chamber (outer cone diameter: $200 \mathrm{~mm}$, inner cone diameter: $120 \mathrm{~mm}$ ) that provided a close to symmetrical flow at sampling head inlets. These sampling heads were chosen as PAS-6 or comparable inhalable dust samplers are often used for stationary and personal measurements in epidemiologic studies. ${ }^{14-17}$ Since wheat allergens may be found predominantly in larger particle-size fractions, ${ }^{18,19}$ the inlet impactor was removed and total flow velocity 


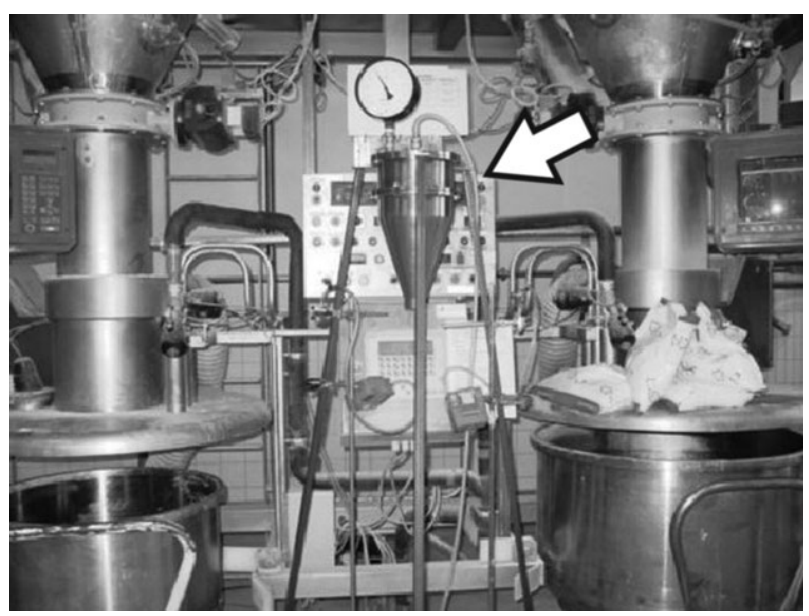

Fig. 1 Experimental setup of the parallel sampling unit (indicated by arrow) near mixing bowls in an industrial bakery.

increased to $40 \mathrm{~L} \mathrm{~min}^{-1}$ to capture particles with a maximal aerodynamic diameter of up to $19 \mu \mathrm{m}$.

Airborne dust samples were collected at 8 locations-bakeries (traditional and industrial) and/or flour mills - in The Netherlands, Germany, Spain and United Kingdom (2 per country). The parallel sampler was situated in the bag-filling area of a flour mill or dough-making area of a bakery, near a known or suspected source of allergen exposure (Fig. 1). Sampling time varied from $30 \mathrm{~min}$ to 6 hours to obtain filters with a range of dust and allergen loads. The sampling heads were equipped with Teflon (PTFE) filters (Milipore, Falp2500), which had been coded and pre-weighed on an analytical balance at IRAS (Institute for Risk Assessment Sciences, Utrecht, The Netherlands) before distribution to the participating institutes. After sampling the filters were returned to IRAS for post-weighing and extraction. During transport and storage before and after weighing, the filters were kept dry in closed cassettes at ambient temperature.

\section{Wheat allergen extraction}

Four major extraction parameters were investigated: elution medium, shaking method, type of tube in which extraction is performed and centrifugation speed at which filters and nondissolved dust components are separated from the extracted proteins. Each parameter had three options ('settings'), based on procedures used thus far by the various partners in the MOCALEX project, or on the results of preliminary pilot experiments:

Elution medium - $2.5 \mathrm{ml}$ PBS (phosphate-buffered saline, pH 7.4), PBS with $0.05 \%$ (v/v) Tween-20 (Merck, Darmstadt, Germany), or PBS with $0.5 \%$ Tween-20;

Shaking method - incubation for $60 \mathrm{~min}$ in an end-over-end rotator, on a high-frequency shaking platform of a laboratory shaker (Gerhardt LS-20), or manual intermittent vortexing $(3 \times 5$ seconds vortexing at 0,30 and 60 minutes);

Tube-Minisorp ${ }^{\mathrm{TM}}(5 \mathrm{ml} ; 12 \times 75 \mathrm{~mm}$, Nunc, Uden, The Netherlands), characterized by polyethylene surfaces with low affinity for proteins, conventional polystyrene tube $(5 \mathrm{ml} ; 12 \times$ $75 \mathrm{~mm}$, Greiner BV, Alphen aan den Rijn, The Netherlands), or a commonly used soda-lime glass tube $(5 \mathrm{ml} ; 12 \times 75 \mathrm{~mm}$, Dispolab, Asten, The Netherlands);

Centrifugation - 15 minutes at either $3000 \mathrm{~g}, 1000 \mathrm{~g}$, or no centrifugation at all.

Duration of extraction was set at $60 \mathrm{~min}$, as preliminary experiments had shown that longer agitation of PTFE filters does not improve wheat allergen recovery, and similar findings have been reported for other occupational allergens. ${ }^{7}$ All extractions were done at ambient temperature, i.e., between 18 and $25{ }^{\circ} \mathrm{C}$, which in 60 minutes is not likely to permit bacterial or fungal growth in the elution medium, especially since dust samples had been stored dry, and in all extractions freshly prepared medium was used.

After centrifugation, $2 \mathrm{ml}$ of fluid was harvested from each sample, divided into small aliquots and stored at $-20{ }^{\circ} \mathrm{C}$ until analyses.

\section{Design}

For the extraction experiments 12 sampling runs were selected from each country, and from each run, 9 of the 10 filters were half-randomly assigned to various extraction procedures. Extraction was performed on 6 days. Each day included elution of 72 filters: 9 filters from 8 runs, 2 runs from 2 different locations in each of the four countries. This resulted after 6 extraction days in a total of 432 extracts.

The half-random assignment was chosen to optimise the use of available filters. The 9 parallel filters from each run were extracted in 9 different ways, such that on each day two extraction parameters were varied, and the other two were kept fixed. When not varied, extraction medium was PBS with $0.05 \%(\mathrm{v} / \mathrm{v})$ Tween-20, shaking method was incubation in an end-over-end-rotator, extraction tube was a conventional polystyrene tube, and centrifugation was performed at $3000 \mathrm{~g}$. For example, on one day the elution medium and shaking method were varied (each with three options), and centrifugation speed and tube choice were kept fixed $(3000 \mathrm{~g}$ and a polystyrene tube); on another day centrifugation speed and elution medium varied, while type of tube (polystyrene) and shaking method (end-over-end rotator) were fixed, etc. Thus, for each extraction parameter, 288 samples were extracted at the default setting, and 72 samples at the two other settings. In this way, all two-way but no higher interactions - which a priori seemed to be rather unlikely-between elution parameters could be assessed. The alternative, a completely random design, would have a high risk of uneven distribution (by chance) of filters over extraction parameter combinations and therefore a lower statistical power.

\section{Storage experiment}

During the MOCALEX project, measured concentrations of some allergens showed some decline after prolonged extract storage at $-20{ }^{\circ} \mathrm{C}$. To explore such storage effects on wheat allergen levels, an additional set of parallel filters was extracted ( $n=54)$ using three elution media and three shaking options (see under wheat allergen extraction). Since in other experiments losses upon storage of another important bakery allergen-fungal $\alpha$-amylase - could partially be prevented by addition of casein), ${ }^{20} 10 \%(\mathrm{v} / \mathrm{v})$ of a $1 \%$ casein solution was 
added to half of the aliquots, before freezing to $-20{ }^{\circ} \mathrm{C}$. Wheat allergens were measured 20 days and 4 months after extraction. Effects of freeze-thaw cycles were excluded by only testing aliquots that had been frozen and thawed once- thus, only the period of storage at $-20{ }^{\circ} \mathrm{C}$ differed.

\section{Determination of wheat allergen concentrations in extracts}

Wheat allergen levels were measured by human IgG4 inhibition EIA, rabbit IgG inhibition EIA and rabbit sandwich EIA. The samples were primarily tested at $1 / 5,1 / 10,1 / 20$ dilutions in the inhibition EIAs, $1 / 100,1 / 300$ and $1 / 900$ in the sandwich EIA, and at higher or lower (lowest 1/2,1/4 and 1/8) dilutions if necessary. The assays are described in detail elsewhere. ${ }^{11}$

\section{Statistical analyses}

Effects of extraction parameters were assessed by mixed regression analysis with 'run' being the random factor, thus allowing adjustment for autocorrelation between samples within a sampling run. Allergen concentrations were ln-transformed before analysis to achieve a normal distribution of data. Two main models were investigated: a model including all results (model $1, n=432$ ) and a model with only results from the 30 runs of which all samples showed detectable values in each assay (model $2, n=270$ ). In model 1 , a value of $2 / 3$ of the lower detection limit of the assay (LOD) was assigned to samples with non-detectable allergen levels. The two modelapproach was chosen to avoid possible underestimation or overestimation of effects, due to either the inclusion of too many samples with results $<$ LOD to which identical values had been assigned, or exclusion of paired observations where one result (from a less effective elution procedure) was $<$ LOD and the other $>$ LOD.

All two-way interactions between parameters (medium-shaking, shaking-tube, tube-centrifugation speed, etc.) were also investigated, but none appeared to contribute significantly to the variance in measured wheat allergen concentrations. Country of sampling was included as a fixed effect to account for possible confounding. Adjustments for dust levels and position of the PAS- 6 sampling head within the parallel sampler did not affect the effect estimates, and were not included in the final analysis.

\section{Results}

Depending on duration of sampling (0.5-6 hours), dust amounts on filters ranged from 0.02 to $6.2 \mathrm{mg}$, and wheat allergen levels from 0.25 to $67.5 \mu \mathrm{g}$. Samples from the UK, Spain, Netherlands and Germany had (geometric) mean dust levels of $0.15,0.23,0.41$ and $0.55 \mathrm{mg} \mathrm{filter}^{-1}$, respectively. These values should not be considered as indicators of real country-dependent differences in exposure levels, and were probably mainly determined by the selection and availability of sampling sites to position the parallel sampler close to wheat dust sources.

Extracts from most of the airborne dust samples $(n=432)$ showed detectable wheat allergen levels in each of the three EIAs. Approximately $86 \%$ of all samples were positive and $5 \%$ negative in all three assays. The highest number of samples $>$ LOD was found with the rabbit IgG sandwich EIA
(94.4\%), while the human and the rabbit inhibition EIAs had about $87 \%$ of the tested extracts positive. Results of the assays correlated well $(r>0.95)$ and showed good agreement in absolute values. ${ }^{11}$

\section{Effects of variations in extraction parameters}

In both regression models the only significant determinant of wheat allergen yield was the choice of elution medium. Extraction in the presence of Tween-20, as shown by each of the three EIAs, was much more effective than extraction in PBS alone, especially for samples with allergen levels $<100 \mathrm{ng} \mathrm{ml}^{-1}$ (Fig. 1A and B). According to the analysis with inclusion of all samples (Table 1a), extractions in PBS resulted in 84\% (rabbit IgG inhibition EIA) to $99 \%$ (rabbit sandwich EIA) lower wheat allergen yields, compared to extraction in PBS with $0.05 \%$ Tween-20. Exclusion of samples with allergen levels <LOD (Table 1b) diminished the effect of elution medium, but it still remained highly significant, with $69 \%$ (rabbit IgG inhibition EIA) to $72 \%$ (rabbit sandwich EIA) lower wheat allergen levels in the absence of Tween. Different Tween concentrations $(0.05 \%$ or $0.5 \%(\mathrm{v} / \mathrm{v}))$ did not cause significant differences in allergen yields. Only in the IgG4 inhibition EIA were slightly lower allergen levels found with $0.5 \%$ than with $0.05 \%$ Tween (Table $1 \mathrm{a}$ and $\mathrm{b})-13.6 \%(p=$ 0.06 , model 1$)$ to $17 \%(p=0.07$, model 2$)$.

The effects of other extraction parameters - shaking method, type of tube and centrifugation speed-were nearly all insignificant, with the effect on allergen yield not exceeding $10 \%$ (Fig. 2). Only when measured with the rabbit IgG inhibition EIA, the use of glass tubes resulted in $14 \%$ (model 1)-15\% (model 2) lower allergen yields compared to the use of polystyrene tube $(p<0.05)$. This effect was not or hardly visible in other two assays (Table 1a and b).

\section{Storage experiment}

Storage of filter extracts for up to four months at $-20{ }^{\circ} \mathrm{C} \mathrm{did}$ not result in significant wheat allergen losses. Median ratios of allergen levels determined at 20 days and at 4 months after the elution were close to 1 (1.0 and 1.27 in the human and the rabbit inhibition EIA, respectively) for extracts containing no casein. In the extracts with added casein, the ratios were 1.17 and 1.04. Thus, no losses (Fig. 3A and B) and no stabilisation effect of casein were observed. However, extracts made in PBS without Tween and with low wheat allergen levels $\left(<10 \mu\right.$ filter $\left.^{-1}\right)$ showed higher allergen concentrations after prolonged storage (Fig. 3C and D).

\section{Discussion}

Different elution methods and immunoassay techniques have been used thus far for wheat airborne allergen exposure measurements ${ }^{14,21-23}$ and no standardised approach is available. Aim of the present study was to assess effects of differences in extraction methods on wheat allergen yields and to suggest an optimised extraction protocol for routine laboratory use. Effects of differences in immunoassay techniques were evaluated and described elsewhere. ${ }^{11}$

A parallel sampling design was used with series of 10 identical dust samples, which would enable evaluation of 
Table 1 ANOVA results for four different extraction parameters, as obtained with the three wheat immunoassays and two statistical models: (a) model 1 (all data included, $n=432$ ); (b) model 2 (restricted data set, $n=270$ ). ANOVA outcomes are given as factors indicating the relative recovery of allergen, compared to the referent parameters $(*)$, together with $95 \%$ confidence intervals (CI)

(a)

\begin{tabular}{|c|c|c|c|c|c|c|}
\hline \multirow[b]{2}{*}{ Effect } & \multicolumn{2}{|c|}{ Human IgG4 inhibition EIA } & \multicolumn{2}{|c|}{ Rabbit IgG inhibition EIA } & \multicolumn{2}{|c|}{ Rabbit polyclonal sandwich EIA } \\
\hline & Factor & CI & Factor & CI & Factor & CI \\
\hline PBS & $0.12^{a}$ & $(0.10-0.14)$ & $0.16^{a}$ & $(0.14-0.18)$ & $0.01^{a}$ & $(0.01-0.01)$ \\
\hline PBS $+0.5 \%$ Tween & $0.86^{c}$ & $(0.74-1.01)$ & 1.01 & $(0.89-1.15)$ & 0.95 & $(0.67-1.36)$ \\
\hline PBS $+0.05 \%$ Tween* & 1 & & 1 & & 1 & \\
\hline Laboratory shaker & 1.06 & $(0.91-1.24)$ & 0.99 & $(0.88-1.12)$ & 1.03 & $(0.72-1.48)$ \\
\hline Intermittent vortexing & 1.10 & $(0.96-1.28)$ & 1.04 & $(0.92-1.18)$ & 1.04 & $(0.73-1.50)$ \\
\hline End-over-end rotator* & 1 & & 1 & $(0.88-1.12)$ & 1 & \\
\hline Glass & 0.99 & $(0.85-1.15)$ & $0.86^{b}$ & $(0.76-0.97)$ & 0.94 & $(0.65-1.34)$ \\
\hline Minisorp & 1.08 & $(0.93-1.26)$ & 1.02 & $(0.90-1.16)$ & 1.00 & $(0.69-1.43)$ \\
\hline Polystyrene* & 1 & & 1 & & 1 & \\
\hline $0 g$ & 1.00 & $(0.86-1.16)$ & 1.01 & $(0.89-1.14)$ & 1.08 & $(0.75-1.55)$ \\
\hline $1000 \mathrm{~g}$ & 1.05 & $(0.91-1.23)$ & 1.05 & $(0.93-1.19)$ & 1.06 & $(0.74-1.51)$ \\
\hline $3000 g^{*}$ & 1 & & 1 & & 1 & \\
\hline
\end{tabular}

(b)

\begin{tabular}{|c|c|c|c|c|c|c|}
\hline \multirow[b]{2}{*}{ Effect } & \multicolumn{2}{|c|}{ Human IgG4 inhibition EIA } & \multicolumn{2}{|c|}{ Rabbit IgG inhibition EIA } & \multicolumn{2}{|c|}{ Rabbit polyclonal sandwich EIA } \\
\hline & Factor & CI & Factor & CI & Factor & $\mathrm{CI}$ \\
\hline PBS & $0.29^{a}$ & $(0.24-0.35)$ & $0.31^{a}$ & $(0.27-0.36)$ & $0.28^{a}$ & $(0.24-0.32)$ \\
\hline PBS + 0.5\% Tween & $0.83^{c}$ & $(0.69-1.02)$ & 1.03 & $(0.89-1.18)$ & 0.90 & $(0.78-1.03)$ \\
\hline PBS $+0.05 \%$ Tween* & 1 & & 1 & & 1 & \\
\hline Laboratory shaker & 1.07 & $(0.93-1.22)$ & 1.02 & $(0.92-1.12)$ & 0.99 & $(0.90-1.09)$ \\
\hline Intermittent vortexing & 1.07 & $(0.94-1.23)$ & 1.01 & $(0.91-1.11)$ & 0.95 & $(0.86-1.04)$ \\
\hline End-over-end rotator* & 1 & & 1 & & 1 & \\
\hline Glass & 0.98 & $(0.86-1.11)$ & $0.85^{b}$ & $(0.77-0.93)$ & $0.91^{c}$ & $(0.83-1.00)$ \\
\hline Minisorp & 1.06 & $(0.93-1.20)$ & 1.04 & $(0.94-1.14)$ & 1.02 & $(0.93-1.12)$ \\
\hline Polystyrene* & 1 & & 1 & & 1 & \\
\hline $0 \mathrm{~g}$ & 1.00 & $(0.87-1.15)$ & 1.04 & $(0.94-1.15)$ & 1.07 & $(0.97-1.18)$ \\
\hline $1000 \mathrm{~g}$ & 1.04 & $(0.91-1.19)$ & 1.05 & $(0.96-1.16)$ & 1.03 & $(0.94-1.14)$ \\
\hline $3000 g^{*}$ & 1 & & 1 & & 1 & \\
\hline
\end{tabular}

${ }^{a}$ Significantly different from the referent parameter, $p<0.0001 .{ }^{b}$ Significantly different from the referent parameter, $0.001<p<0.05 .{ }^{c}$ On the borderline of statistical significance, $0.05<p<0.1$.

different extraction options. Of the investigated parameters, the elution medium (PBS with or without $0.05 \%$ or $0.5 \%$ Tween-20) appeared to be the most important: the presence of Tween-20 resulted in a three- to 100 -fold increase in wheat allergen recovery. The effect was strongly concentration-dependent and much larger at lower allergen levels. The effect estimates depended slightly on the type of wheat assay and the statistical model, but were not essentially different. Similar effects of Tween-20 with significantly improved recovery of eluted allergens or other agents have been found for rodent urinary allergens, ${ }^{7,9}$ outdoor allergens ${ }^{24}$ and endotoxins, ${ }^{25}$ but not for airborne potato antigens. ${ }^{26}$ Reason for these discrepancies may be that Tween-20 facilitates the release of partially hydrophobic agents, like LPS (i.e., endotoxins) and
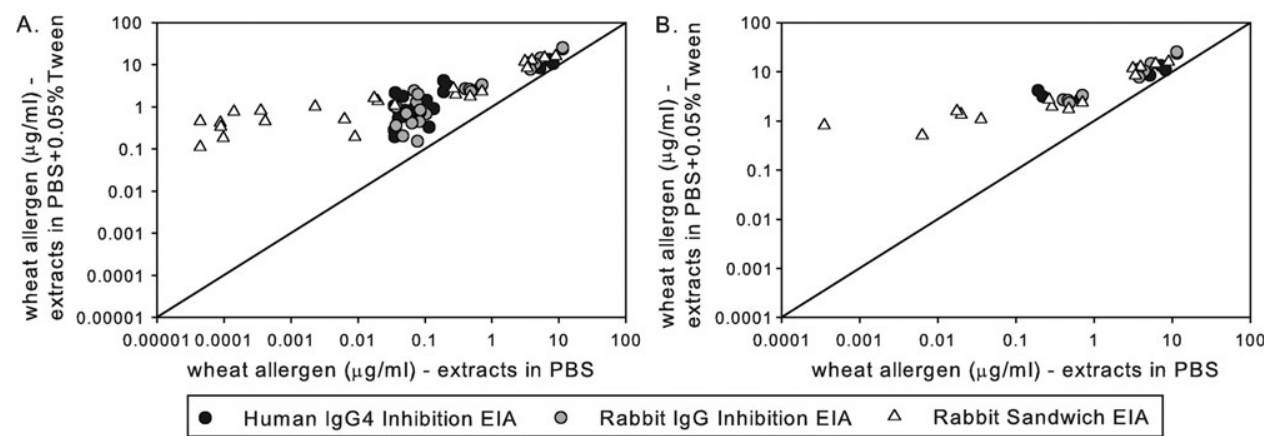

Fig. 2 Effect of Tween-20 in elution medium $(0.05 \%(\mathrm{v} / \mathrm{v}))$ on wheat allergen yields, with all samples (A) included, or only with samples with levels $>$ LOD (B), as measured by the human IgG4 inhibition EIA, rabbit IgG inhibition EIA and rabbit sandwich EIA. The allergen levels are given as average values from the 24 parallel sampling runs where filters were eluted with varying media. Three filters of each run were eluted in PBS, and three in PBS $+0.05 \%$ Tween-20. Line in the figure represents the line of unity. 

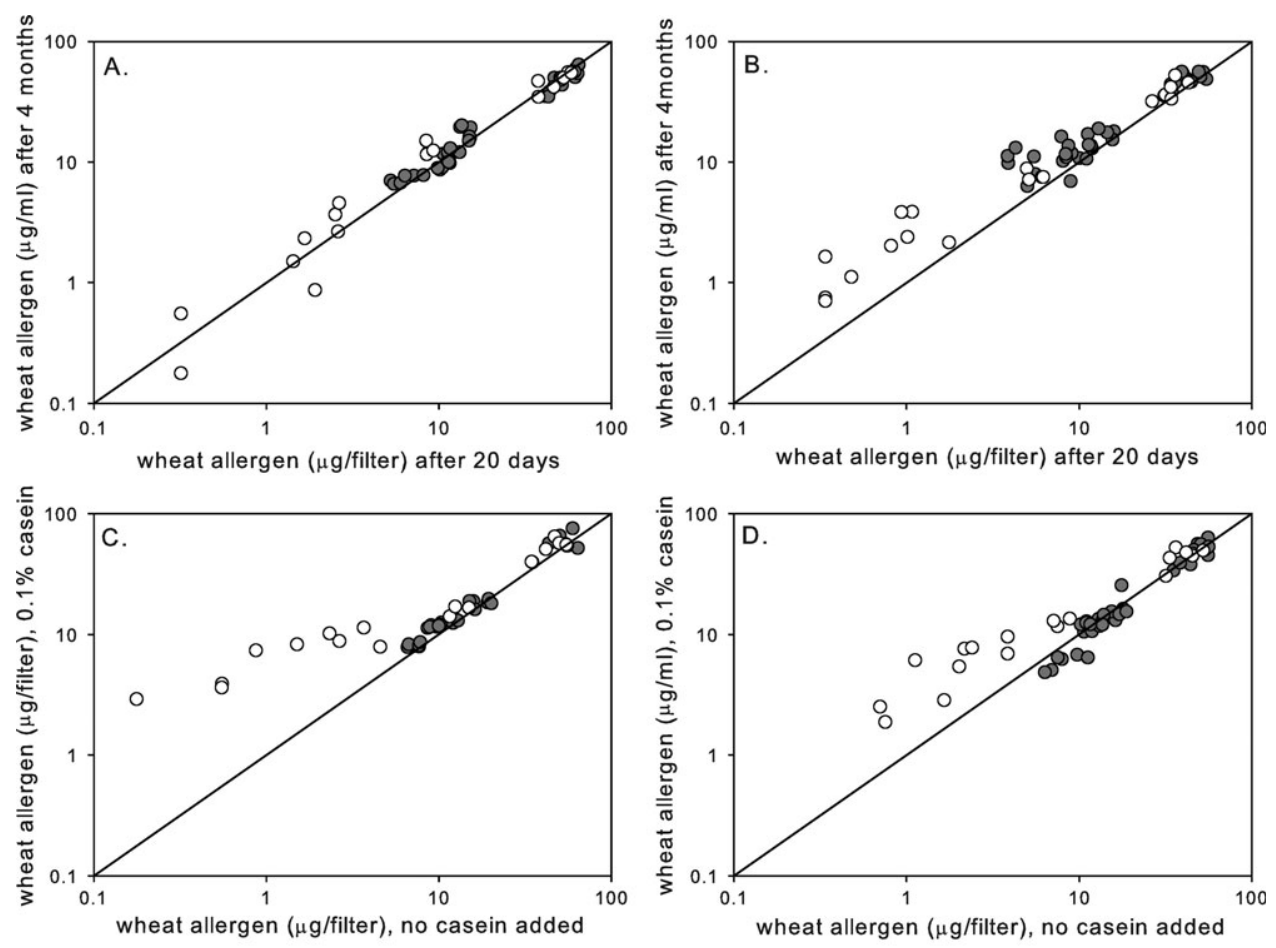

- PBS-Tween extracts $O$ PBS extracts

Fig. 3 Wheat allergen yields after 20 days and after 4 months of extract storage without any stabilizer, as measured by the human IgG4 inhibition EIA (A); Wheat allergen yields after 20 days and after 4 months of extract storage without any stabilizer, as measured by the rabbit IgG inhibition EIA (B); Effects of casein on wheat allergen yields after 4 months of extract storage, as measured by the human IgG4 inhibition EIA (C); Effects of casein on wheat allergen yields after 4 months of extract storage, as measured by the rabbit IgG inhibition EIA (D).

possibly some of the wheat flour proteins, while the effect for already highly water-soluble proteins from potato tubers ${ }^{26,27}$ is much less pronounced. Another reason may be the difference in the type of filters used in these studies. In some of the studies glass fiber filters were used, and in the others, including our study, PTFE filters. The structure of glass fiber filter may allow deeper penetration of allergens into filter pores, and in these cases Tween-20 might improve allergen release. The hydrophobic PTFE filters are, on the other hand, likely to retain protein molecules on the filter surface. ${ }^{7}$ Thus, the effect of Tween-20 in this study may be primarily due to an increased wettability of Teflon filters by detergent-containing medium, and more effective release of proteins from the non-dissolvable wheat flour matrix. Tween-20 may also increase recoveries by preventing protein losses due to non-specific binding of proteins to vial walls or pipette tips.

Higher concentrations of Tween in elution medium did not lead to increased allergen recovery, as found by Jensen and coworkers, ${ }^{24}$ who showed a dose-dependent effect of Tween-20 on the elution of Timothy grass pollen allergens from glass fibre filters. In fact, we found a tendency for higher wheat allergen yields with $0.05 \%(\mathrm{v} / \mathrm{v})$ than with $0.5 \%$ Tween- 20 . Different types of filters used in these two studies might offer an explanation - higher Tween-20 concentrations may facilitate elution from inner structures of glass fibre filters, while not further enhancing elution of proteins from the wheat flour matrix and/or a Teflon filter surface.
The shaking method, type of tube and centrifugation speed had no or only minor effects on allergen yields. Use of glass tubes resulted in somewhat lower allergen yields, compared to conventional polystyrene tubes, especially when measured in the rabbit IgG inhibition EIA (up to $15 \%$ lower yields). Since working with glass always implicates enhanced risk of injury, and yields were not higher with the more expensive Minisorp ${ }^{\mathrm{TM}}$ tube with low protein-binding affinity, a conventional polystyrene tube may be recommended for routine use.

Inclusion of a centrifugation step after extraction had, surprisingly, no significant effect on allergen yields. In fact, we expected interference in the EIA by undissolved flour matrix particles in non-centrifuged extracts. Such interference may be more likely with heavily loaded filters (e.g., $>10 \mathrm{mg}$ dust), and would cause imprecision and poor reproducibility of EIA results. Since dust levels in that range were not found in our study, but are not exceptional in routine measurements in bakeries, we recommend inclusion of centrifugation step after extraction, with a speed of $1000 \mathrm{~g}$ that should be sufficient to avoid problems in the EIAs.

Wheat allergen levels in extracts were stable upon storage for at least 4 months at $-20{ }^{\circ} \mathrm{C}$, and no stabilisation effect of casein was found. The effects of casein were investigated since it had been found to prevent losses of another important bakery allergen that is often measured in the same airborne dust samples - fungal $\alpha$-amylase. ${ }^{20}$ Although no major interference of casein with the measured wheat allergen levels was 
observed, at low allergen levels it showed a tendency to increase allergen yields. This effect was not due to crossreactivity of casein with the anti-wheat antibodies, as found in preliminary experiments (data not shown), and it was observed practically only in extracts made in PBS without Tween-20. Thus, in the absence of Tween-20, casein seems to take over its role in preventing allergen losses.

\section{Conclusion}

Addition of Tween-20 is essential for an optimal elution of wheat allergens from airborne flour dust samples, especially at lower allergen levels. Other, less stringent recommendations comprise the use of ordinary polystyrene tubes as extraction vials, a relatively simple type of shaking method, and lowspeed centrifugation after extraction. Filter extracts can be stored for at least 4 months at $-20{ }^{\circ} \mathrm{C}$ with no apparent allergen losses as far as wheat allergens are concerned. An optimal extraction and extract storage procedure should, however, account for effects on levels of other occupational allergens if these have to be measured in the same extracts, like fungal $\alpha$-amylase in case of wheat allergens.

\section{Acknowledgements}

The authors would like to thank Prof. Peter S. Thorne for critical reading of the manuscript. This study was supported by the European Community 5th framework 'Quality of life' programme (project QLK-CT-2001-00432) (MOCALEX).

\section{References}

1 G. Block, K. S. Tse, K. Kijek, H. Chan and M. Chan-Yeung, Clin. Allergy, 1983, 13, 359-370.

2 M. G. Prichard, G. Ryan and A. W. Musk, Br. J. Ind. Med., 1984, 41, 450-454.

3 R. Sutton, J. H. Skerritt, B. A. Baldo and C. W. Wrigley, Clin. Allergy, 1984, 14, 93-107.

4 W. Weiss, C. Vogelmeier and A. Görg, Electrophoresis, 1993, 14, 805-816.

5 R. Houba, D. Heederik and G. Doekes, Am. J. Respir. Crit. Care Med., 1998, 158, 1499-1503.
6 L. Lillienberg, X. Baur, G. Doekes, L. Belin, M. Raulf-Heimsoth, I. Sander, A. Ståhl, J. Thissen and D. Heederik, Ann. Occup. Hyg., 2000, 44, 427-433.

7 S. Gordon, R. D. Tee, D. Lowson and A. J. Newman Taylor, Ann. Occup. Hyg., 1992, 36, 575-587.

8 A. Renström, S. Gordon, P. H. Larsson, R. D. Tee, A. J. Newman Taylor and P. Malmberg, Clin. Exp. Allergy, 1997, 26, 1314-1321.

9 A. Renström, S. Gordon, A. Hollander, J. Spithoven, P. H. Larsson, K. M. Venables, D. Heederik and P. Malmberg, Allergy, 1999, 54, 150-157.

10 A. Hollander, S. Gordon, A. Renström, J. Thissen, G. Doekes, P. H. Larsson, P. Malmberg, K. M. Venables and D. Heederik, Allergy, 1999, 54, 142-149.

11 J. Bogdanovic, I. M. Wouters, I. Sander, M. Raulf-Heimsoth, J. Elms, M. J. Rodrigo, D. J. J. Heederik and G. Doekes, Clin. Exp. Allergy, 2006, 39(9), in press.

12 W. Eduard, G. Blomquist, B. Herbert Nielsen and K. Kulvik Heldal, Ann. Occup. Hyg., 2001, 45, 493-498.

13 W. M. ter Kuile, Staub-Reinhalt. Luft, 1984, 44, 211-216.

14 R. Houba, P. van Run, D. J. J. Heederik and G. Doekes, Clin. Exp. Allergy, 1996, 26, 154-163.

15 R. Houba, P. van Run, G. Doekes, D. Heederik and J. Spithoven, J. Allergy Clin. Immunol., 1997, 99, 286-292.

16 P. Bulat, K. Myny, L. Braeckman, M. van Sprundel, E. Kusters, G. Doekes, K. Possel, J. Droste and M. Vanhoorne, Ann. Occup. Hyg., 2004, 48, 57-63.

17 C. Peretz, N. de Pater, J. de Monchy, J. Oostenbrink and D. Heederik, Scand. J. Work Environ. Health, 2005, 31, 65-74.

18 C. P. Sandiford, M. J. Nieuwenhuijsen, R. D. Tee and A. J. Taylor, Allergy, 1994, 49, 891-893.

19 J. Bogdanovic, A. J. de Pater, G. Doekes, I. M. Wouters and D. J. J. Heederik, Ann. Occup. Hyg., 2006, 50, 131-136.

20 I. Sander, I. Sander, E. Zahradnik, J. Bogdanovic, M. RaulfHeimsoth, I. M. Wouters, A. Renström, J. Harris-Roberts, E. Robinson, M. J. Rodrigo, N. Goldscheid, T. Brüning and G. Doekes, submitted.

21 R. D. Tee, D. J. Gordon, S. Gordon, B. Crook, A. J. Nunn, A. W. Musk, K. M. Venables and A. J. Taylor, Br. J. Ind. Med., 1992, 49, 581-587.

22 C. P. Sandiford, M. J. Nieuwenhuijsen, R. D. Tee and A. J. Newman Taylor, Clin. Exp. Allergy, 1994, 24, 450-456.

23 K. Wiley, M. M. Smith, L. J. Allan and P. Griffin, Int. Arch. Allergy Immunol., 1997, 114, 278-284.

24 J. Jensen, L. K. Poulsen, K. Mygind, E. R. Weeke and B. Weeke, Allergy, 1989, 44, 52-59.

25 J. Douwes, P. Versloot, A. Hollander, D. Heederik and G. Doekes, Appl. Environ. Microbiol., 1995, 61, 1763-1769.

26 J. P. Zock, A. Hollander, G. Doekes and D. Heederik, Am. Ind. Hyg. Assoc. J., 1996, 57, 567-570.

27 S. Gorinstein, S. Yamagata and D. Hadziyev, J. Food Biochem., 1988, 12, 37-49. 